Tohoku J. Exp. Med., 2006, 209, 311-320

\title{
Fluvastatin Alters Psychomotor Performance and Daily Activity but not the Spatial Memory in Rats
}

\author{
Sukrucan H. Baytan, Mehmet Alkanat, Mehmet Ozeren, ${ }^{1}$ Murat Ekincl ${ }^{2}$ and \\ Ahmet Akgun \\ Department of Physiology, ${ }^{1}$ Department of Obstetrics and Gynecology, \\ Karadeniz Technical University, Medical School, Trabzon, Turkey, and \\ ${ }^{2}$ Department of Computer Engineering, Engineering School, Karadeniz, \\ Technical University, Trabzon, Turkey
}

\begin{abstract}
Baytan, S.H., Alkanat, M., Ozeren, M., Ekinci, M. and Akgun, A. Fluvastatin Alters Psychomotor Performance and Daily Activity but not the Spatial Memory in Rats. Tohoku J. Exp. Med., 2006, 209 (4), 311-320 — Statins, inhibitors of cholesterol synthesis for treating dyslipidemia and preventing cardiovascular complications, have been shown to alter central nervous system functions. Our aim was to investigate the effects of the fluvastatin, a member of statin family, on psychomotor performance, daily activity and spatial memory. Sprague-Dawley rats were treated with fluvastatin $(n=8)$ or placebo as a control $(n=11)$ regardless of sex. Fluvastatin $(7.5 \mathrm{mg} / \mathrm{kg})$ was administered orally once a day for four weeks, while the control group was administered only placebo. Psychomotor performance was measured by rotarod tests. No significant difference was observed in the fluvastatin group over the course of weeks, but the control group preferred to stay on the device shorter times $(p<0.05)$. For the first three weeks of the drug administration there was a statistical difference between the groups, however no difference was found after the 4th week. There was no difference in the Barnes maze spatial memory test between the groups and also within the groups over the course of time. Daily activity tests revealed that stereotypical and vertical movements of the fluvastatin group were significantly less than the control group in all four weeks. Ambulatory movements and the distances taken by the fluvastatin group were decreased significantly over the course of time $(p<0.005$ and $p<0.001$, respectively), but the control group did not reveal any significant change. Our results suggest that fluvastatin altered psychomotor performance and daily activity in rats, but it did not affect the spatial memory. These behavioral changes might be associated with alterations in the composition of the brain lipids caused by fluvastatin. statin; fluvastatin; behavior; Barnes maze; psychomotor
\end{abstract}

(C) 2006 Tohoku University Medical Press

Statins, inhibitors of the 3-hydroxy-3-methylglutaryl-CoA (HMG-CoA) reductase in the synthesis of cholesterol, are widely used in the prevention of cardiovascular diseases by decreasing blood lipid levels (Maron et al. 2000; Collins et al. 2004). Statins change lipid metabolism, lower low-density lipoproteins, increase high-density lipoproteins, and decrease tryglicerids depending on specific statin used (Schaefer et al. 2004).

Cholesterol metabolism and transport of

Received February 22, 2006; revision accepted for publication May 25, 2006.

Correspondence: Dr. Sukrucan H. Baytan, KTU Tip Fak, Fizyoloji ABD, 61080 Trabzon, Turkey.

e-mail: cbaytan@gmail.com 
cholesterol turnover within the brain were reviewed by Bjorkhem and Meaney (2004). Statin applications and lowered plasma cholesterol levels were found to affect central nervous system (CNS) functions in humans. Severe irritability and aggression with statin usage in six patients were reported by Golomb et al. (2004a, b). They also addressed that there is a need for more data to establish the impact of hydrophilic and lipophilic statins on cognition, aggression, and brain serotonin activity. Cultured glial cells have HMGCoA reductase as the major rate-limiting enzyme for their synthesis of cholesterol (Snipes and Suter 1997). A well known fact is that myelination is preceded by increased esterification of cholesterol, which is possibly a mechanism for accumulation of the required very high amounts of cholesterol in the developing CNS (Norton and Cammer 1984). Experimental evidence for a cholesterol shuttle from astrocytes to neurons in an in vitro system had been presented by Mauch et al. (2001). Benefical effects of atorvastatin on human higher cortical functions were studied by Parale et al. (2006), which lays strong foundations for future human studies about mental activities and statin usage relationships. These behavioral studies clearly showed that statin treatment can alter behavior with its possible cholesterol altering effects in the CNS.

The effects of several statins on lipid composition of the brain in rats, including fluvastatin, were observed by Vecka et al. (2004), in which fluvastatin, an important member of the statin family, was shown to increase sphingomyelin and to decrease diphosphatidylglycerol contents in rat brains. Yet, the most important changes in the fatty acid profile being in the ceramide monohexosides, lead to a decrease in the content of saturated fatty acids and an increase in the content of polyunsaturated fatty acids. Moreover, it has been shown that chronic administration of statins alters multiple gene expression patterns in the mouse cerebral cortex (Johnson-Anuna et al. 2005). From these studies' standpoints, changes in CNS functions and behaviors could be expected with statin treatment.

In this study, we investigated the effects of fluvastatin on behavioral changes by evaluating spatial memory, psychomotor performance and daily activity in Sprague-Dawley rats.

\section{Methods}

\section{General procedure}

The study was approved by Karadeniz Technical University, Medical School animal ethics committee (12-October-2005 ref: 473).

Twelve male and twelve female Sprague-Dawley rats with initial weights of 200-240 g, 5-6 months old were provided and maintained in our vivarium (constant temperature of $23 \pm 1{ }^{\circ} \mathrm{C} ; 60 \pm 10 \%$ relative humidity; 12-h/12-h light cycle; food and water ad lib.). The animals were kept in our vivarium for two weeks for habituation without any testing and training. The animals were divided into two groups (6 males and 6 females in each group total of 12 animals in each group) as control and fluvastatin groups using the random table. Fluvastatin $\left(\right.$ Lescol $^{\mathrm{TM}}$, Novartis, Basel, Switzerland) was obtained commercially and homogenized in phosphate buffered saline (PBS). The control group was administered the PBS solution without the drug as placebo. The administered solution volume each time was $0.5 \mathrm{ml}$ in both groups. Fluvastatin $(7.5 \mathrm{mg} / \mathrm{kg}$ once a day $)$ and placebo was administered for a total of 28 days orally by gavage around 8:30 am. Testing was designed as all measurements were taken once a week on the 0th, 7th, 14th, 21st, and 28th days, regardless of sex. Five animals had to be excluded from the study during the testing period, two animals died from unknown causes, two animals repeatedly failed to find the goal box in the Barnes maze, and one animal was observed to be pregnant. With the excluded animals, the fluvastatin group consisted of 8 animals ( 4 males and 4 females). The control group consisted of 11 (5 males and 6 females) animals for statistical evaluation.

\section{Rotarod test (Psychomotor performance)}

An accelerating rotarod device designed for mice and rats was used for the psychomotor performance evaluation. The rod was $4.5 \mathrm{~cm}$ in diameter, covered with non-slippery material, $30 \mathrm{~cm}$ above the device floor (Commat Inc., Ankara, Turkey). The animals were first habituated on the rod and trained for 5 days before testings had started. Each training day the animals were expected to stay on the rod as long as they could. After the first fall, it was taken to its homecage to rest for at least $15 \mathrm{~min}$ and then the second trial was started. Every 
animal took three trials per day during the training period. The training trials started with the stationary rod for $60 \mathrm{sec}$, and then it started to revolve and gradually increase to $16 \mathrm{rpm}$. There was no punishment system that was used on the rotarod tests. After the training period and one day resting period, the testing phase was started. In the testing phase the same procedure was applied, except each animal underwent 5 trials in each experiment day. Latency times are measured in seconds.

Barnes maze test (Spatial memory and reference learning)

A modified Barnes maze was utilized to perform spatial learning and reference memory tests described by Barnes (1979) and Turner et al. (2004). The maze was $122 \mathrm{~cm}$ in diameter made of water resistant white material, had 12 holes with a diameter of $9.5 \mathrm{~cm}$ equidistant from each other, and $3 \mathrm{~cm}$ away from the perimeter. Height of the maze was $140 \mathrm{~cm}$ to discourage animals from jumping to the floor. A $10 \mathrm{~cm}$ wide, $20 \mathrm{~cm}$ long, and $12 \mathrm{~cm}$ high stainless steel goal box was placed under one hole during the training and experiment sessions. Animals were placed in a light-tight start box placed in the middle of the maze, equivalent to the goal box. Extra maze cues were placed around the maze, big round and square dark colored posters hung on the white wall, and a divider curtain was used to hide the experimenters while the tests were being performed. A floodlight was used $180 \mathrm{~cm}$ over the maze for aversive reaction on tests. A video system, which was used for recording animal movements, was attached on top of the maze. A television monitor was located behind the divider curtain. The video recordings were evaluated offline to calculate the distance taken by the animals. Distance measurements were calculated by using the Software developed by and the Department of Computer Science and the Department of Physiology, Medical School in Karadeniz University.

Before the tests began, all animals were allowed to stay on the Barnes maze for one hour moving freely without the goal box for habituation. Afterwards, 4 training sessions for 5 days were conducted. Each animal was brought to the testing laboratory separately. After a five minute resting period, it was placed under the start box and kept there for $10 \mathrm{sec}$ with their heads always positioned to the north. Lights were turned on, experimenters moved behind the divider, and then the start box was removed remotely by a pulley system and the animals were expected to find the goal box. When the animal found the escape hole, the lights were turned off, the escape hole was covered by a light tight material and the animal was allowed to stay there for $60 \mathrm{sec}$. The procedure was repeated four times during each testing period. If an animal could not find the hole in $5 \mathrm{~min}$, an experimenter gently drove the animal to the escape hole and the animal was allowed to rest there for $60 \mathrm{sec}$. In the training and testing trials, the goal box position remained the same between measurements, but maze holes were turned randomly each time for animals to avoid following odor trails. Before and after each test and training session, the maze was wiped clean for the same purpose as well. The animals were also trained once before each experiment day and the hole position was changed for each testing week. In the first training trial, animals did not know the position of the goal box. In the testing period, the hole remained in the same position. The testing procedure was the same as in the training period, but when the start box was removed, a timer was started. Head pokes or closely approaching and visually checking the wrong holes by the animals were counted as errors. Repeated pokes in the same hole or several stares to a wrong hole in a single approach were counted as one error. The timer stopped when all paws touched the goal box. Four measurements were recorded on each testing day.

\section{Daily activity}

A computerized cage system for animal activity was used to assess 24-hr daily locomotor activity, such as ambulatory movements, vertical movements, stereotypical movements, and distances were taken in centimeters (Commat Inc.). The cage was a clear cube (each side 42 $\mathrm{cm})$. Horizontal infrared beam rays on each four sides $(2.5 \mathrm{~cm}$ apart to each other, $2.5 \mathrm{~cm}$ in height) monitored the horizontal movements. There was also a similar beam design in the height of $13 \mathrm{~cm}$ to record vertical movements of the animal. Stereotypical movements such as itching, sniffing and head bobbings were recorded. Also the numbers of horizontal movements in the cage (ambulatory) were counted, while stereotypical or vertical movements were excluded in the ambulatory counts. Vertical movements, and the total horizontal distances taken by the animals (distance) were calculated.

The data within the control and fluvastatin groups in the proceeding weeks were statistically evaluated with the Friedman test. These groups were compared with each other separately every week from week zero through week four by Mann-Whitney's U-test. A $p$-value of smaller than $0.05(p<0.05)$ was considered to be 
significant. Statistical analysis was performed by statistical program package, SPSS version 13.01 (Lead Technologies Inc., Chicago, IL, USA).

\section{RESULTS}

\section{Rotarod test}

The fluvastatin group showed no significant difference over the course of the weeks while the control group stayed on the rotarod device for shorter times over the course of time $(p<0.05$, Table 1). As expected, there were no differences observed in the 0th week. While after the 1st, 2nd and 3rd weeks a difference was found, there was no difference observed after the 4th week.

\section{Barnes maze test}

There was no difference between the fluvastatin and the control group for errors, latency times and distances $(p>0.05$, Table 2). Also, comparisons between the fluvastatin and placebo groups for four weeks indicated there was no significant difference between the groups.

\section{Daily activity tests}

All data about daily activity tests are shown in the Table 3.

TABLE 1. Latency times of fluvastatin and control groups on the rotarod device $\S$.

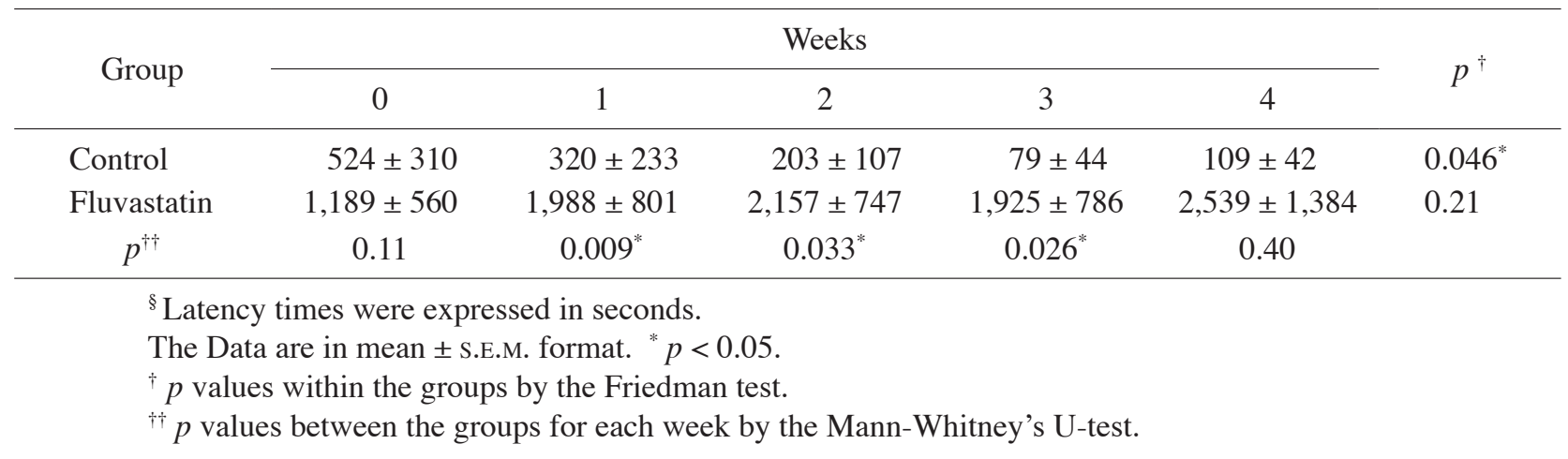

TABlE 2. Barnes maze error counts, latency times and distances for each week in the groups ${ }^{\S}$.

\begin{tabular}{|c|c|c|c|c|c|c|c|}
\hline & \multirow{2}{*}{ Group } & \multicolumn{5}{|c|}{ Weeks } & \multirow{2}{*}{$p^{\dagger}$} \\
\hline & & 0 & 1 & 2 & 3 & 4 & \\
\hline \multirow[t]{3}{*}{ Error counts } & Control & $11 \pm 3$ & $8 \pm 1$ & $8 \pm 2$ & $5 \pm 1$ & $8 \pm 1$ & 0.23 \\
\hline & Fluvastatin & $8 \pm 1$ & $7 \pm 1$ & $10 \pm 3$ & $8 \pm 2$ & $8 \pm 2$ & 0.81 \\
\hline & $p^{\dagger \dagger}$ & 0.60 & 0.40 & 0.84 & 0.21 & 0.78 & \\
\hline \multirow[t]{3}{*}{ Latency (sec) } & Control & $77 \pm 35$ & $49 \pm 12$ & $64 \pm 34$ & $37 \pm 11$ & $55 \pm 12$ & 0.62 \\
\hline & Fluvastatin & $41 \pm 9$ & $31 \pm 7$ & $48 \pm 15$ & $43 \pm 13$ & $40 \pm 9$ & 0.64 \\
\hline & $p^{\dagger \dagger}$ & 0.40 & 0.15 & 0.78 & 0.97 & 0.21 & \\
\hline \multirow[t]{3}{*}{ Distance $(\mathrm{cm})$} & Control & $264 \pm 27$ & $234 \pm 66$ & $293 \pm 98$ & $202 \pm 54$ & $256 \pm 51$ & 0.68 \\
\hline & Fluvastatin & $260 \pm 18$ & $299 \pm 67$ & $292 \pm 76$ & $261 \pm 60$ & $216 \pm 32$ & 0.19 \\
\hline & $p^{\dagger \dagger}$ & 0.78 & 0.40 & 0.66 & 0.49 & 0.97 & \\
\hline
\end{tabular}

${ }^{\S}$ Error counts on the Barnes maze, latency times for entering right hole in seconds, and distances taken on the maze in centimeters.

The Data are in mean \pm S.E.M. format.

${ }^{\dagger} p$ values within the groups by the Friedman test.

${ }^{\dagger} p$ values between the groups for each week by the Mann-Whitney's U-test. 
TABLE 3. Daily activity measurements for fluvastatin and control groups ${ }^{\S}$.

\begin{tabular}{|c|c|c|c|c|c|c|c|}
\hline & \multirow{2}{*}{ Group } & \multicolumn{5}{|c|}{ Weeks } & \multirow{2}{*}{$p^{\dagger}$} \\
\hline & & 0 & 1 & 2 & 3 & 4 & \\
\hline \multirow[t]{3}{*}{ Stereotypical } & Control & $10,580 \pm 974$ & $11,707 \pm 890$ & $11,382 \pm 1,013$ & $11,432 \pm 884$ & $11,344 \pm 914$ & 0.59 \\
\hline & Fluvastatin & $8,568 \pm 593$ & $8,595 \pm 619$ & $7,953 \pm 603$ & $8,213 \pm 564$ & $7,925 \pm 601$ & 0.99 \\
\hline & $p^{\dagger \dagger}$ & 0.16 & $0.017^{*}$ & $0.006^{*}$ & $0.017^{*}$ & $0.004^{*}$ & \\
\hline \multirow[t]{3}{*}{ Ambulatory } & Control & $3,767 \pm 301$ & $3,871 \pm 452$ & $4,043 \pm 474$ & $4,124 \pm 445$ & $3,648 \pm 608$ & 0.36 \\
\hline & Fluvastatin & $4,141 \pm 360$ & $3,285 \pm 244$ & $3,168 \pm 251$ & $2,674 \pm 264$ & $2,920 \pm 398$ & $0.002^{*}$ \\
\hline & $p^{\dagger \dagger}$ & 0.41 & 0.22 & 0.07 & $0.005^{*}$ & 0.06 & \\
\hline \multirow[t]{3}{*}{ Vertical } & Control & $381 \pm 54$ & $438 \pm 40$ & $503 \pm 53$ & $467 \pm 43$ & $475 \pm 59$ & 0.45 \\
\hline & Fluvastatin & $385 \pm 52$ & $258 \pm 32$ & $213 \pm 31$ & $201 \pm 31$ & $226 \pm 51$ & 0.08 \\
\hline & $p^{\dagger \dagger}$ & 0.62 & $0.003^{*}$ & $0.001^{*}$ & $0.001^{*}$ & $0.006^{*}$ & \\
\hline \multirow[t]{3}{*}{ Distance } & Control & $8,434 \pm 734$ & $8,259 \pm 1,032$ & $8,639 \pm 1,209$ & $8,939 \pm 1,437$ & $8,514 \pm 1,319$ & 0.91 \\
\hline & Fluvastatin & $9,609 \pm 883$ & $8,149 \pm 623$ & $6,957 \pm 697$ & $5,954 \pm 594$ & $6,722 \pm 1,048$ & $0.000^{*}$ \\
\hline & $p^{\dagger \dagger}$ & 0.28 & 0.74 & 0.28 & 0.07 & 0.06 & \\
\hline
\end{tabular}

$\S$ Counts of stereotypical, ambulatory, vertical and horizontal movements, and distances taken in centimeters, in the daily activity cage.

The Data are in mean \pm S.E.M. format. ${ }^{*} p<0.05$.

${ }^{\dagger} p$ values within the groups by the Friedman test.

${ }^{\dagger \dagger} p$ values between the groups for each week by the Mann-Whitney's U-test.

Stereotypical movements. During the course of time no difference was shown statistically in either group. When the difference between groups was analyzed each week, the fluvastatin group was found to make less stereotypical movements from the 1 st week to 4 th week.

Ambulatory movements. The fluvastatin group was found to have less ambulatory movement counts during the course of time $(p<0.005)$, but the same significance was not observed for the control group. When the difference between the groups was investigated on a weekly basis, the fluvastatin group was found to have less ambulatory movements only on the 3rd week.

Vertical movements. During the course of time no difference was observed statistically in either group. When the difference between the groups was investigated for each week, the fluvastatin group was found to have less vertical movements from the 1 st week to 4 th week.

Total distance. The fluvastatin group was found to take less distance during the course of time $(p<0.001)$, but no change was observed in the control group. When the difference between groups over the weeks was investigated, no statistical difference between the groups was observed.

From the results, psychomotor performance was found to be altered in the control group during the course of the time that they ran on the rotarod device for shorter time periods. Between the 1 st and the 3 rd weeks, a significant difference was observed between the control and fluvastatin groups. But, the fluvastatin group did not change their psychomotor performance during the course of the time. Spatial memory was not affected by fluvastatin use, according to the Barnes maze measurements. The results of daily activity tests revealed that the fluvastatin group made less ambulatory movements and took less distances during the course of time. When compared to the control group, the fluvastatin group displayed stereotypical and vertical movements that were 
found to be significantly less in the last four drug administered weeks.

\section{Discussion}

It has been suggested that statins may provide beneficial effects that are not limited to reduced levels of low-density lipoproteins and triglycerides in the blood (Liao 2002, 2004, 2005). Statins are expected to affect any organ or system involved in lipid metabolism, including the CNS, which has a concentration of unesterified cholesterol higher than any other tissue $(23 \mathrm{mg} / \mathrm{g})$, containing $23 \%$ of the sterol present in the whole body pool in humans while accounting for only $2.1 \%$ of body weight. The relationships between plasma cholesterol concentration and sterol metabolism in the CNS or cognitive functions in the brain remain unclear (Dietschy and Turley 2004). There are reports of the effects of statins on cognitive functions in patients with primary hypercholesterolemia (Gengo et al. 1995). Another study suggested that lovastatin treatment might cause small performance decrements on neuropsychological tests of attention and psychomotor speeds, but in the same study no evidence was found in psychological distress or substantial cognitive function alterations in hypercholesterolemic adults. Another study, comprising of 308 hypercholesterolemic adults, suggested that there are minor decrements in cognitive functioning with simvastatin treatment (Muldoon et al. 2000, 2004). However, two cases were reported to have significant temporary cognitive impairment related to statin therapy (King et al. 2003). A review of a literature study about statin-associated memory loss of 60 cases using Med-Watch drug surveillance system of the Food and Drug Administration between November 1997 and February 2002 was presented by Wagstaff et al. (2003). Severe irritability and aggression with statin usage in six patients were also reported by Golomb et al. (2004a). There is an ongoing double-blind, placebo-controlled clinical trial with selected 1,000 non-cardiac patients that will be available for assessing the impact of the statins in cognitive functions, personality, sleep, behavioral irritability, and blood serotonin levels correlated with aggression (Golomb et al. 2004b).

Fluvastatin is known as a lipophilic drug (De Angelis 2004). Effects of fluvastatin in the rat brain were shown by Vecka et al. (2004). Another study reported that lovastatin and simvastatin strongly reduced the levels of free cholesterol in synaptozomal plazma membranes and lovastatin and pravastatin significantly reduced cholesterol levels in the exofacial membrane leaflet. These changes were accompanied by modified membrane bulk fluidity.

Statins reduce the expression of the raft marker protein flotillin. Statins directly or indirectly exert various effects on cell membrane cholesterol homeostasis in the CNS (Kirsch et al. 2003). A rat brain perfusion study demonstrated that fluvastatin crosses blood brain barrier with permeability coefficient of $2.5 \times 10(-4)$ (Guillot et al. 1993). Some other statins cross the blood brain barrier to a much greater extent. These studies suggest that statins affect brain lipid composition, cell membrane and cholesterol homeostasis; hence CNS functions cause behavioral alterations.

Fluvastatin usage did not affect the spatial memory and reference learning in the rats in our study. Even though a study indicated that Atorvastatin treatment promoted the restoration of spatial memory function in rats with traumatic brain injury, this might be a result of increased blood flow after the injury ( $\mathrm{Lu}$ et al. 2004). Existing reports are not sufficient to make clear conclusions about the memory effects of the statin usage in animals and humans. The effects of statin administration on memory and learning in rats are less than clear.

Our results on daily activity tests that the fluvastatin group made less movements during the course of time compared to the control group suggested that these results may be related to a muscle injury caused by fluvastatin use. It was shown that fluvastatin inhibits Rho/Rho-kinase signalling and causes disruption of the actin cytoskeleton in rat smooth muscle cultures (Kato et al. 2004). In some cases, side effects occur in skeletal muscle, including myositis or even rhabdomyolysis. Simvastatin causes apoptosis in differentiated 
human skeletal muscle cells (Sacher et al. 2005). The potential role of the depleted isoprenoid pool in the pathophysiology of statin myopathy is discussed by Baker (2005).

If a muscle injury was present with fluvastatin treatment, we would expect less activity and shorter walking time from the fluvastatin group in the rotarod tests. But the fluvastatin group did not show this type of behavior and preferred to stay and walk on rotarod for periods of longer time. These results suggest that fluvastatin may alter psychomotor behavior at higher cortical or spinal levels. With applied rotarod device tests, anxiety may be elicited by stimuli associated with the omission or loss of reward. Since neither reward nor a punisment is employed during tests, this might invoke a state of anxiety state in the subjects and activate the Behavioral Inhibition System (BIS) proposed by Gray (1991).

The Behavioral Approach System (BAS) activates approach behaviors in response to cues for reward or non-punishment (Gray 1994). It may be neurophysiologically associated with the motor programming systems in the CNS. The key components are the basal ganglia, the dopaminergic fibers that ascend from the mesencephalon to innervate the basal ganglia, thalamic nuclei closely linked to the basal ganglia and similarly neocortical areas such as motor, sensorimotor, and prefrontal cortex which are also closely linked to the basal ganglia. Dopamine is known to play an essential moderating role in the functioning of the BAS (Depue and Iacono 1989).

BIS is the main cortical behavioral inhibitory system. Signals of punishment, non-reward, novel stimuli, and innate fear stimuli lead to behavioral inhibition, an increment in tense arousal, and increased attention. The BIS may be considered both as a cognitive and physiological system (Fowles 1988; Gray 1991). Cognitively, the role of the BIS is to compare the current state of the world with expectations and to inhibit and modify behavior that leads to deviations from expectation. Physiologically, the comparator function of the BIS is associated with the septohippocampal system. Input to this system comes from the prefrontal cortex output flows through the noradrenergic fibers of the locus coeruleus, and serotonergic fibers from the median raphe (Gray 1994).

According to Gray (1990), the hippocampus is an important contributor to the BIS, causing the individual to stop and attend to environmental cues forwarded from other brain structures. Information processed by the hippocampus is joined in the hypothalamus with information processed by a brain structure called the septum. The hypothalamus generates behavioral responses. The role of the amygdala in this system is to give emotional response based on conditioning to stimuli that predict the occurrence of reinforcement. The BIS is in part moderated through the action of the neurotransmitter Gammaaminobutyric acid (GABA) on the ascending noradrenergic and serotonergic pathways to the hippocampus. GABA is mostly inhibitory and it is involved in the regulation of behavior. Rats demonstrated a weakened emotional response after the hippocampus was lesioned, and GABA increases in the nucleus accumbens were blocked during the acquisition and expression of a conditioned emotional response (Saul'skaia and Gorbachevskaia 1998). Increases in GABA levels in the amygdala mitigate the intensity of anxiety. GABAergic neurons are modulated by dopaminergic and noradrenergic input from the nucleus accumbens (Steiniger-Brach and Kretchmer 2005). Once BIS was activated, predictions were made based on memories of previous experience provided by the prefrontal cortex. The memorybased predictions were then compared with events occurring in the present. Incongruence between the prediction and reality results in behavioral inhibition and increased physical arousal and attention, the individual searches for more information (Gray 1994).

Dopamine and acetylcholine were used in the nucleus accumbens according to Gray (1990). In the BAS the nucleus accumbens is involved in general incentive motivation for approach behavior. The path between the nucleus accumbens and the ventral tegmental area of the brain (where dopamine is present) allows motive to be translated to behavior through dopaminergic relay of 
input to the prefrontal cortex as presented by Lee et al. (1998).

It has been reported that high doses of simvastatin upregulate dopamine D1 and D2 receptor expression in the rat prefrontal cortex by a possible involvement of endothelial nitric oxide synthase (Wang et al. 2005). Protective effects of fluvastatin from the 1-methyl-4-phenylpyridine induced hydroxyl radical generation in the rat striatum in cultured cells is reported (Obata and Yamanaka 2000).

From our results we can speculate that fluvastatin treated rats ran longer times on the rotarod caused by either prefrontal cortical neuron loss from the BIS related prefrontal association areas resulted in decreased BIS activity or increased BAS activity lead by increased cortical dopaminergic activity, which might be the cause of the decreased inhibited reaction towards a stressful stimulus. There is a possibility that these mechanisms might have been worked together.

BAS or BIS is not in effect in daily activity evaluations, because there is no reward or punishment stimulus involved in this type of test. Atorvastatin attenuated the glutamate-induced increase of intracellular calcium, which was associated with a modulation of $\mathrm{N}$-methyl-D-asparate (NMDA) receptor functions (Boesel et al. 2005). Also it is shown that HMG-CoA reductase inhibition causes a profound reduction of neurite length, neurite loss and neuron death in undifferentiated rat cortical neurons (Schulz et al. 2004). So, it could be expected that modulation of NMDA dependent glutamate receptors and cortical neuron deaths and neurite losses can lead a functional change in all those systems, including behavioral response systems.

Our results have shown that stereotypical and vertical movements of daily activities were less in the fluvastatin group than the control group. But ambulatory movements and distances taken in a day is decreased within the fluvastatin group during the course of time. Ambulatory movements and distances were not found different between the control and fluvastatin groups, with the exception of the 3rd week comparison between the groups. Animal reduced activity can be either or both results of loss of motor cortical neurons and possible muscle damage by high dose of fluvastatin treatment. This is caused by apoptosis in differentiated human skeletal muscle cells (Sacher et al. 2005) or statin affected NMDA receptor functions. Local dopaminergic modulation of the motor activity induced by NMDA receptor stimulation in the ventral hippocampus was shown by Gimenez-Llort (2002).

Our results suggest that fluvastatin might affect psychomotor performance and daily activity in rats, but not the spatial memory. We conclude that unreduced pscyhomotor performance in the fluvastatin group in contrast to the control group may be associated with altered BIS and BAS under the effects of the fluvastatin in the brain. Daily activity changes are suggested to be results of the reduced dopaminergic activity along with cortical lipid profile changes or with possible muscle damage of the fluvastatin. Further behavioral and receptor level neural studies are needed to explain these effects.

\section{Acknowledgments}

Special thanks to Ozturk Medical Inc., Trabzon-Turkey for the financial support of this study.

We also like to thank Mr. Kemal Uzun for his enormous helpful efforts in the studies.

\section{References}

Baker, S.K. (2005) Molecular clues into the pathogenesis of statin-mediated muscle toxicity. Muscle Nerve, 31, 572-580.

Barnes, C.A. (1979) Memory deficits associated with senescence: a neurophysiological and behavioral study in the rat. J. Comp. Physiol. Psychol., 93, 74-104.

Bjorkhem, I. \& Meaney, S. (2004) Brain cholesterol: long secret life behind a barrier. Arterioscler. Thromb. Vasc. Biol., 24, 806-815.

Boesel, J., Gandor, F., Harms, C., Synowitz, M., Harms, U., Djoufack, P.C., Megow, D., Dirnagl, U., Hortnagl, H., Fink, K.B. \& Endres, M. (2005) Neuroprotective effects of atorvastatin against glutamate-induced excitotoxicity in primary cortical neurones. J. Neurochem., 92, 1386-1398.

Collins, R., Armitage, J., Parish, S., Sleight, P. \& Peto, R. (2004) Effects of cholesterol-lowering with simvastatin on stroke and other major vascular events in 20536 people with cerebrovascular disease or other high-risk conditions. Lancet, 363, 757-767.

De Angelis, G. (2004) The influence of statin characteristics on their safety and tolerability. Int. J. Clin. Pract., 58 , 945-955.

Depue, R.A. \& Iacono, W.G. (1989) Neurobehavioral aspects 
of affective disorders. Annu. Rev. Psychol., 40, 457-492.

Dietschy, J.M. \& Turley, S.D. (2004) Thematic Review Series: Brain Lipids. Cholesterol metabolism in the central nervous system during early development and in the mature animal. J. Lipid Res., 45, 1375-1397.

Fowles, D.C. (1988) Psychophysiology and psychopathology: A motivational approach. Psychophysiology, 25, 373-391.

Gengo, F., Cwudzinski, D., Kinkel, P., Block, G., Stauffer, L. \& Lines, C. (1995) Effects of treatment with lovastatin and pravastatin on daytime cognitive performance. Clin. Cardiol., 18, 209-214.

Gimenez-Llort, L., Wang, F., Ogren, S.O. \& Ferre, S. (2002) Local dopaminergic modulation of the motor activity induced by N-methyl-D-aspartate receptor stimulation in the ventral hippocampus. Neuropsychopharmacology, 26, 737-743.

Golomb, B.A., Kane, T. \& Dimsdale, J.E. (2004a) Severe irritability associated with statin cholesterol-lowering drugs. QJM, 97, 229-235.

Golomb, B.A., Criqui, M.H., White, H.L. \& Dimsdale, J.E. (2004b) The UCSD Statin Study: a randomized controlled trial assessing the impact of statins on selected noncardiac outcomes. Control. Clin. Trials., 25, 178-202.

Gray, J.A. (1990) Brain systems that mediate both emotion and cognition. Cognition and Emotion, 4, 269-288.

Gray, J.A. (1991) Neural systems, emotion, and personality. In: Neurobiology of Learning, Emotion, and Affect, edited by J. Madden, Raven Press, NewYork, pp. 273-306.

Gray, J.A. (1994) Framework for a taxonomy of psychiatric disorder. In: Emotions: Essays on Emotion Theory, edited by S.H.M. van Goozen, N.E. van de Poll \& J. Sergeant, Hillsdale Erlbaum, New Jersey, pp. 29-59.

Guillot, F., Misslin, P. \& Lemaire, M. (1993) Comparison of fluvastatin and lovastatin blood-brain barrier transfer using in vitro and in vivo methods. J. Cardiovasc. Pharmacol., 21, 339-346.

Johnson-Anuna, L.N., Eckert, G.P., Keller, J.H., Igbavboa, U., Franke, C., Fechner, T., Schubert-Zsilavecz, M., Karas, M., Muller, W.E. \& Wood, W.G. (2005) Chronic administration of statins alters multiple gene expression patterns in mouse cerebral cortex. J. Pharmacol. Exp. Ther., 312, 786-793.

Kato, T., Hashikabe, H., Iwata, C., Akimoto, K. \& Hattori, Y. (2004) Statin blocks Rho/Rho-kinase signalling and disrupts the actin cytoskeleton: relationship to enhancement of LPS-mediated nitric oxide synthesis in vascular smooth muscle cells. Biochim. Biophys. Acta, 1689, 267-272.

King, D.S., Wilburn, A.J., Wofford, M.R., Harrell, T.K., Lindley, B.J. \& Jones, D.W. (2003) Cognitive impairment associated with atorvastatin and simvastatin. Pharmacotherapy, 23, 1663-1667.

Kirsch, C., Eckert, G.P. \& Mueller, W.E. (2003) Statin effects on cholesterol micro-domains in brain plasma membranes. Biochem. Pharmacol., 65, 843-856.

Lee, R.S., Koob, G.F. \& Henriksen, S.J. (1998) Electrophysiological responses of nucleus accumbens neurons to novelty stimuli and exploratory behavior in the awake, unrestrained rat. Brain Res., 20, 317-322.

Liao, J.K. (2002) Lipid lowering: the role of statins in vascular protection. Int. J. Cardiol., 86, 5-18.

Liao, J.K. (2004) Statins: potent vascular anti-inflammatory agents. Int. J. Clin. Pract. Suppl., 143, 41-48.

Liao, J.K. (2005) Effects of statins on 3-hydroxy-3-methylglutaryl coenzyme a reductase inhibition beyond low-density lipoprotein cholesterol. Am. J. Cardiol., 96, 24F-33F.

Lu, D., Mahmood, A., Goussev, A., Schallert, T., Qu, C., Zhang, Z.G., Li, Y., Lu, M. \& Chopp, M. (2004) Atorvastatin reduction of intravascular thrombosis, increase in cerebral microvascular patency and integrity, and enhancement of spatial learning in rats subjected to traumatic brain injury. $J$. Neurosurg., 101, 813-821.

Maron, D.J., Fazio, S. \& Linton, M.F. (2000) Current perspectives on statins. Circulation, 101, 207-213.

Mauch, D.H., Nagler, K., Schumacher, S., Goritz, C., Muller, E.C., Otto, A. \& Pfrieger, F.W. (2001) CNS synaptogenesis promoted by glia-derived cholesterol. Science, 294, 1354-1357.

Muldoon, M.F., Barger, S.D., Ryan, C.M., Flory, J.D., Lehoczky, J.P., Matthews, K.A. \& Manuck, S.B. (2000) Effects of lovastatin on cognitive function and psychological well-being. Am. J. Med., 108, 538-546.

Muldoon, M.F., Ryan, C.M., Sereika, S.M., Flory, J.D. \& Manuck, S.B. (2004) Randomized trial of the effects of simvastatin on cognitive functioning in hypercholesterolemic adults. Am. J. Med., 117, 823-829.

Norton, W.T. \& Cammer, W. (1984) Isolation and characterization of myelin. In: Myelin, edited by P. Morell, Vol. 2, Plenum Press, New York, pp. 147-180.

Obata, T. \& Yamanaka, Y. (2000) Protective effect of fluvastatin, a new inhibitor of 3-hydroxy-3-methylglutaryl coenzyme A reductase, on MPP(+)-induced hydroxyl radical in the rat striatum. Brain Res., 860, 166-169.

Parale, G.P., Bahetti N.N., Kulkarni, P.M. \& Panchhal, N.V. (2006) Effects of atorvastatin on higher functions. Eur. J. Clin. Pharmacol., 27, 425-446.

Sacher, J., Weigl, L., Werner, M., Szegedi, C. \& Hohenegger, M. (2005) Delineation of myotoxicity induced by 3-hydroxy3-methylglutaryl CoA reductase inhibitors in human skeletal muscle cells. J. Pharmacol. Exp. Ther., 314, 1032-1041.

Saul'skaia, N.B. \& Gorbachevskaia, A.I. (1998) Participation of the hippocampus in regulation of GABA release in the nucleus accumbens during emotional conditioning. Ross Fiziol. Zh. Im. I. M. Sechenova., 84, 43-49.

Schaefer, E.J., McNamara, J.R., Tayler, T., Daly, J.A., Gleason, J.L., Seman, L.J., Ferrari, A. \& Rubenstein, J.J. (2004) Comparisons of effects of statins (atorvastatin, fluvastatin, lovastatin, pravastatin, and simvastatin) on fasting and postprandial lipoproteins in patients with coronary heart disease versus control subjects. Am. J. Cardiol., 93, 31-39.

Schulz, J.G., Bosel, J., Stoeckel, M., Megow, D., Dirnagl, U. \& Endres, M. (2004) HMG-CoA reductase inhibition causes neurite loss by interfering with geranylgeranylpyrophospha te synthesis. J. Neurochem., 89, 24-32.

Snipes, G.J. \& Suter, U. (1997) Cholesterol and Myelin. In: Subcellular Biochemistry, edited by R. Bittman, Vol. 28, Plenum Press, New York, pp. 173-204.

Steiniger-Brach, B. \& Kretschmer, B.D. (2005) Different function of pedunculopontine GABA and glutamate receptors in nucleus accumbens dopamine, pedunculopontine glutamate and operant discriminative behavior. Eur. J. Neurosci., 22, 1720-1730.

Turner, R.J., Van den Heuvel, C. \& Vink, R. (2004) Amiloride increases neuronal damage after traumatic brain injury in rats. J. Am. Coll. Nutr., 23, 534S-537S.

Vecka, M., Tvrzicka, E., Stankova, B., Novak, F., Novakova, O. \& Zak, A. (2004) Hypolipidemic drugs can change the composition of rat brain lipids. Tohoku J. Exp. Med., 204, 299-308. 
Wagstaff, L.R., Mitton, M.W., Arvik, B.M. \& Doraiswamy, P.M. (2003) Statin-associated memory loss: analysis of 60 case reports and review of the literature. Pharmacotherapy, 23, 871-880.

Wang, Q., Ting, W.L., Yang, H. \& Wong, P.T. (2005) High doses of simvastatin upregulate dopamine D1 and D2 receptor expression in the rat prefrontal cortex: possible involvement of endothelial nitric oxide synthase. $B r . J$. Pharmacol., 144, 933-939. 